\title{
Role of gga-miR-221 and gga-miR-222 during Tumour Formation in Chickens Infected by Subgroup J Avian Leukosis Virus
}

\author{
Zhenkai Dai ${ }^{1,+}$, Jun Ji ${ }^{2,+}$, Yiming Yan ${ }^{1}$, Wencheng Lin ${ }^{1}$, Hongxin Li ${ }^{1,3}$, Feng Chen ${ }^{1,3,4,5}$, \\ Yang Liu ${ }^{1}$, Weiguo Chen ${ }^{1,4,5}$, Yingzuo Bi ${ }^{1,4,5}$ and Qingmei Xie ${ }^{1,2,3,4,5, *}$ \\ Received: 13 October 2015; Accepted: 2 December 2015; Published: 11 December 2015 \\ Academic Editor: Andrew Mehle \\ 1 College of Animal Science, South China Agricultural University \& Guangdong Provincial Key Lab of \\ Agro-Animal Genomics and Molecular Breeding, Guangzhou 510642, China; \\ daizhenkai@stu.scau.edu.cn (Z.D.); wuliaozhiji404@163.com (Y.Y.); Linwencheng@cau.edu.cn (W.L.); \\ lihongxing12@126.com (H.L.); fengch@scau.edu.cn (F.C.); 15813372565@163.coml (Y.L.); \\ Wgchen81@scau.edu.cn (W.C.); yizbi@126.com (Y.B.) \\ 2 China-UK-NYNU-RRes Joint laboratory of Insect Biology, Nanyang Normal Universiy, Nanyang 473000, \\ China; jijun84@gmail.com \\ 3 Institute of Animal Science, Guangdong Academy of Agriculture Sciences, Guangzhou 510640, China \\ 4 Key Laboratory of Animal Health Aquaculture and Environmental Control, Guangzhou 510642, China \\ 5 South China Collaborative Innovation Center for Poultry Disease Control and Product Safety, \\ Guangzhou 510640, China \\ * Correspondence: qmx@scau.edu.cn; Tel.: +20-8528-0279 \\ + These authors contributed equally to this work.
}

\begin{abstract}
Subgroup J avian leukosis virus (ALV-J) causes a neoplastic disease in infected chickens. Differential expression patterns of microRNAs (miRNAs) are closely related to the formation and growth of tumors. (1) Background: This study was undertaken to understand how miRNAs might be related to tumor growth during ALV-J infection. We chose to characterize the effects of miR-221 and miR-222 on cell proliferation, migration, and apoptosis based on previous microarray data. (2) Methods: In vivo, the expression levels of miR-221 and miR-222 were significantly increased in the liver of ALV-J infected chickens $(p<0.01)$. Over-expression of gga-miR-221 and gga-miR-222 promoted the proliferation, migration, and growth of DF-1 cells, and decreased the expression of BCL-2 modifying factor (BMF) making cells more resistant to apoptosis. (3) Results: Our results suggest that gga-miR-221 and gga-miR-222 may be tumour formation relevant gene in chicken that promote proliferation, migration, and growth of cancer cells, and inhibit apoptosis. BMF expression was significantly reduced in vivo 70 days after ALV-J infection. They may also play a pivotal role in tumorigenesis during ALV-J infection.
\end{abstract}

Keywords: subgroup J avian leukosis virus; gga-miR-221; gga-miR-222; BMF; apoptosis; cancer; cisplatin; doxorubicin

\section{Introduction}

The subgroup J Avian Leukosis virus (ALV-J) can form tumors on chickens and can thus lead to their death; it also can cause non-tumor diseases, namely the secondary infection caused by immunosuppression [1]. The symptoms are emaciation, anemia, tolerance viremia, and immunosuppression, all of which are able to seriously affect poultry production [2,3]. ALV-J has affected the poultry industry worldwide, with the exception of New Zealand. It has been detected in America [4], the Middle East [5], Australia [6], and has caused substantial economic losses. 
Tumors are a kind of disease of poly-genetic abnormality and regulatory disorder. The forming mechanism of it is that carcinogenic factor activates the division of normal cells, breaks the limit of the cell cycle [7] and inhibits apoptosis [8]. This will gradually turn these normal cells into cancer cells which are able to automatically and abnormally proliferate with astonishing speed, and transfer to and invade other organs through the circulation system. Apoptosis is a conserved form of cell death that plays a pivotal role in the formation of cells and the stability of tissues. If apoptosis becomes dysfunctional, it can lead to cancer and autoimmunity. Apoptosis can be triggered through several different pathways. Apoptosis is regulated in part by the BCL-2 family of proteins. The BCL-2 family contains both apoptosis-inhibitors and apoptosis-inducers that act to maintain the stability, structure, and function of mitochondria [9-12]. BCL-2 modifying factor (BMF) is an apoptosis-inducing member of the BCL-2 family in the bcl-2 homology domain only proteins (BH3-only) sub-family, named for having only one characteristic $\mathrm{BH} 3$ protein domain [13]. The $\mathrm{BH} 3$-only proteins likely activate other apoptosis effectors, like Bax and Bak. Activation may occur directly, for example, Bim, Bid [14,15], and p53-upregulated mediator of apoptosis (PUMA) [16] bind to Bax forming oligomers that induce apoptosis; or indirectly, for example, PUMA [17], Bad, and BMF [18] bind to apoptosis-inhibitors (BCL-2 or BCL-x1) and replace them with apoptosis-inducers (Bax and Bak). Chicken bmf can induce apoptosis after transfection in Michigan Cancer Foundation-7(MCF7) and HeLa cell lines [19]. In a mouse model, absence of a single Bim allele exacerbates leukemia [20], and Bim deficiency can accelerate the development of cancer by allowing cells to survive [21]. Thus, the BH3-only proteins are recognized as tumor inhibitors.

MicroRNA (miRNA, miR) is a non-coding single-stranded RNA molecule approximately [22] base pairs in length that regulates gene expression [22,23]. Calin et al. [24] were the first to provide evidence of the essential role of miRNA disorders in the process of tumor-formation. MiR-15 and miR-16 are located in the 13q14 chromosome region, the partial absence of which was strongly influential in an outbreak of chronic lymphocytic leukemia (CLL). In addition, miR-15 and miR-16 are negatively correlated with expression of the anti-apoptotic gene BCL-2 [25], which inhibits apoptosis at the level of the mitochondria and is critical for cancer cells [26]. In poultry, Li et al. [27], have reported gga-miR-375 may function as a tumor suppressor in infection of ALV-J.

MiR-221 and miR-222, are examples of miRNA that is highly conserved among chordates and is located on the $X$ chromosome in humans, rats, and mice [28]. In chickens, miR-221 and miR-222 are located on chromosome 1 . Our previous study has shown that gga-miR-221 and gga-miR-222 to be frequently up-regulated in the livers of chickens 10 weeks post ALV-J infection [29]. Increased expression of these miRNAs has been extensively documented in many different types of tumors including nervous system leukemia [30,31], breast cancer [32], prostate cancer [33] and gastric cancer [34]. Given the over-expression levels of miR-221 and miR-222 in terminal malignant tumors, they are likely therapeutic targets for carcinoma. Galardi et al. [35], suggested that the regulatory factor of cell cycle p27 (Kip l) may be a target of miR-221 and miR-222 regulation, and found that the quantity of p27 in pancreas cells was negatively correlated with the expression level of miR-221 and miR-222, suggesting they likely regulate cell proliferation and the cell cycle. Inhibition of miR-221 and miR-222 could possibly inhibit growth and induce apoptosis in cells [36]. Gramantieri et al. [37], demonstrated that BMF is a target of miR-221 and may play a role in mediating miR-221-induced inhibition of apoptosis in hepatocellular carcinoma. Lambeth et al. [38], have reported increased expression of Gallus gallus (gga)-miR-221 and gga-miR-222 in poultry in the cancer cell line MSB-1 transformed by MDV (Marek's Disease Virus), suggesting a link between oncogenic poultry viruses and expression levels of miR-221 and miR-222. They also provided evidence that gga-miR-221 and gga-miR-222 inhibit p27 (Kip 1). Despite the link to increased expression of miR-221 and miR-222 in MDV infection, there has not been a comprehensive study assessing gga-miR-221 and gga-miR-222 expression in chickens infected by ALV-J.

To understand more fully whether there is a correlation between differential expression of gga-miR-221 and gga-miR-222 and ALV-J pathogenesis, we examined the functions of gga-miR-221 
and gga-miR-222 in cell proliferation, migration, and apoptosis in vitro, and determined the expression levels of gga-miR-221 and gga-miR-222 in ALV-J infection in vivo.

\section{Materials and Methods}

\subsection{Virus and Cell Lines}

The NX0101 strain of ALV-J was graciously provided by Cui Zhizhong from Shangdong Agricultural University, People's Republic of China. It was isolated from a parent breeder farm of white meat-type in 2001 [39]. The DF-1 was an immortalized chicken embryo fibroblast cell line, and was obtained from American Type Culture Collection (Manassas, VA, USA). The DF-1 cells were stored in Dulbecco's modified eagle medium (DMEM) supplemented with $10 \%$ fetal bovine serum (FBS).

\subsection{Cell Transfections}

DF-1 cells were transfected with miRNA mimics for gga-miR-221, gga-miR-222 (Shanghai GenePharma Co., Ltd., Shanghai, China) or Negative control mimics (named gga-miR-NC; designed as described previously) using X-tremeGENE siRNA Transfection Reagent (Roche Applied Science, Mannheim, Germany) as the transfection reagent. For each transfection, $40 \mathrm{nM}$ of miRNA mimics were respectively used in a six-well plate, unless otherwise indicated. Non-transfected cells were used as the blank control (mock group).

\subsection{Cell Proliferation Assay}

DF-1 cells were grown in DMEM supplemented with 10\% FBS in 96-well plates at $1.0 \times 10^{4}$ cells/well. Twenty-four hours after the inoculation, the DF-1 cells were divided into four equal groups. MiRNA mimics for gga-miR-221, gga-miR-222, and the NC mimics were transfected into DF-1 cells in triplicate at $40 \mathrm{nM}$. The fourth group of mock transfected DF-1 cells were established in parallel as a control. The DF-1 cells were stained with $10 \mu \mathrm{L}$ of WST-1 (cell proliferation dye) 24,48 , and $72 \mathrm{~h}$ after transfection, incubated for $2 \mathrm{~h}$ at $37^{\circ} \mathrm{C}$, and then the absorbance was read at $450 \mathrm{~nm}$ using a multimode microplate reader.

\subsection{Colony Formation Assay}

DF-1 cells were grown in 24-well plates at $1.6 \times 10^{5}$ cells/well. Twenty-four hours after plating, gga-miR-221, gga-miR-222, and negative control mimics were transfected into the DF-1 cells in triplicate. A fourth mock transfected group was established in parallel as a control. To separate the cells, pancreatin was added $24 \mathrm{~h}$ after the transfection. The DF-1 cells were seeded in six-well plates at 1000 cells/well, and grown in DMEM $+10 \%$ FBS for two weeks. The DMEM was replaced every three days. At the end of two weeks, the cells were washed with PBS and immobilized on the plates for 15 min through using methyl alcohol. After the methyl alcohol was removed, the cells were stained with $0.1 \%$ crystal violet for $15 \mathrm{~min}$, and then washed three times with PBS. The plates were photographed to determine the number of colonies.

\subsection{Wound Healing Assay}

Gga-miR-221, gga-miR-222, and normal control (NC) mimics transfected into DF-1 cells in triplicate in 24-well plates at $1.6 \times 10^{5}$ cells/well. A mock transfected group was established in parallel as a control. Twenty-four hours after the transfection, a $200 \mathrm{uL}$ yellow pipette tip was used to scratch the cells growing on the plates, even pressure was applied throughout, to create a "wound" in vitro. Unattached cells were removed by washing twice with complete medium. The cells were photographed under a microscope at 0,24 , and $48 \mathrm{~h}$ post wounding. 


\subsection{Analysis of Cell Apoptosis}

The cell apoptosis index was determined using AnnexinV-FITC/Propidium iodide (PI) staining and DAPI to identify nuclei. DF-1 cells were transfected with the gga-miR-221, gga-miR-222, and NC mimics in six-well plates at $2 \times 10^{5}$ cells/well. A mock transfected group was established in parallel as a control. Twenty-four hours after transfection, the cells were serum starved for $48 \mathrm{~h}$, treated with cisplatin $(0.5 \mu \mathrm{g} / \mathrm{mL})$ for $72 \mathrm{~h}$, or treated with doxorubicin $(0.2 \mu \mathrm{g} / \mathrm{mL})$ for $72 \mathrm{~h}$. Annexin V-FITC/PI apoptosis assay was conducted using the Annexin V-FITC/PI apoptosis assay kit (Beyotime Insititute of Biotechnology, Shanghai, China) according to the manufacturer's instructions. Cell morphology was observed utilizing fluorescence microscope and a nuclear DAPI (Beyotime Insititute of Biotechnology) stain. The rate of apoptosis was determined using the following formula: apoptosis rate $(\%)=$ number of apoptotic cells/ total cell number (the total cell number is equal to or greater than 500).

\subsection{Vector Construction}

To construct luciferase reporter vectors, pmiRGLO-BMF-3'UTR-wt and pmiRGLOBMF-3'UTR-mut, wild-type $3^{\prime}$ UTR fragment of BMF were amplified by RT-PCR using the primers $5^{\prime}$-GT TCTCGAGGGAGCACTGAACAAACACT- $3^{\prime}$ and $5^{\prime}$-GTGGTCTAGACACGAAGCAAATATACTG CACA- $3^{\prime}$.mutant-type of BMF were amplified by RT-PCR using the primers $5^{\prime}$-AACCTGCATG TCTCACTGGGTCTCC-3' ${ }^{\prime}$ and $5^{\prime}$-GAGAGGACGTACTTTTGTTTATCCTGGTT- $3^{\prime}$. The amplified putative binding sites for miR221 and miR-222 in the $3^{\prime}$ UTR sequence were inserted downstream of the stop codon of firefly luciferase in the pmiR-GLO Dual-Luciferase miRNA Target Expression Vector (Ambion, Promega, Beijing, China). The 3'UTR-pmiR-GLO recombinant plasmids were identified by $\mathrm{PCR}$ and restriction enzyme analysis, and then selected for further sequencing.

\subsection{Luciferase Activity Assay}

DF-1 cells were grown in 24 -well plate at $3.4 \times 10^{5}$ cells/well. The cells reached $80 \%-90 \%$ confluence after $24 \mathrm{~h}$. The DF-1 cells were transfected $10 \mathrm{nmol} / \mathrm{L}$ gga-miR-221 and gga-miR-222 mimics 20 ng BMF-3'UTR-wt, or BMF-3'UTR-mut, and 4 ng pRL-TK (Promega). Luciferase activity was determined $48 \mathrm{~h}$ after the transfection using the Dual-Glo ${ }^{\circledR}$ Luciferase Assay System kit according to the manufacturer's instructions.

\subsection{Western Blot}

Gga-miR-221, gga-miR-222, and NC mimics were transfected into DF-1 cells in triplicate in 24-well plates at $1.6 \times 10^{5}$ cells/well. A mock transfected group was established in parallel as a control. The cells were lysed and the protein extracted $48 \mathrm{~h}$ after the transfection. BMF was detected using a Rabbit anti-Human BMF polyclonal antibody (1:1000; Abcam, Cambridge, UK) and a goat anti-rabbit IgG-HRP (1:5000; Proteintech Group, Inc., Chicago, IL, USA). The loading control was $\beta$-actin (1:600; predicted molecular weight: $42 \mathrm{kDa}$; Proteintech Group, Inc.).

\subsection{Animal Infection Assay}

One-day-old SPF pullets $(n=200)$ were randomly divided into a control group and an infection group. The pullets in the control group were inoculated with an equivalent volume of sterilized normal saline in the enterocoelia, and the pullets in the infected group received ALV-J NX0101 at $10^{3.7}$ tissu culture infective dose $\mathrm{TCID}_{50}$ in $0.2 \mathrm{~mL}$. Both of the groups were fed in a negative pressure isolator, and were offered food and water Ad libitum. During the course of the experimental infection, 100 chickens were infected with no deaths reported. All animals were euthanized and autopsied upon completion of the experiments. At the early stage of infection, chickens in the infection group were as healthy as those in the control group. After 40 day post infection, chickens in the infected group presented symptoms of anemia, such as inappetence, pale comb, cooling-down of chicken feet as well as progressive emaciation. Compared to the chickens in control group, Chickens in the infected group 
had histopathological changes, such as hepatomegaly and splenomegaly. Beginning at 10-days old, the pullets $(n=6)$ were weighed every 10 days and sacrificed for testing. Morbidity was observed and documented. Tissue samples were obtained from the liver, spleen, marrow, and blood and stored at $-70{ }^{\circ} \mathrm{C}$ until miRNA and mRNA extraction. The experiment lasted 90 days and nine samples were obtained. Use of animals in this study was approved by the South China Agricultural University Committee of Animal Experiments (approval ID 201004152) (Guangzhou, China).

\subsection{Real-Time Quantitative RT-PCR}

Total RNA was extracted from the liver, spleen, and blood samples taken from the pullets in the control and infected groups at each time point using TRIZOL (Invitrogen, Carlsbad, CA, USA). The miRNA was extracted using Isolation of small and large RNA (Macherey-Nagel, Berlin, Germany).

The relative expression levels of gga-miR221, gga-miR-222, bmf by RT-qPCR. Gga-miR221 and gga-miR-222 expression was normalized to the 5s snRNA (chicken), and Bmf were normalized to HMBS (chicken). gga-miR221 and gga-miR-222 were amplified by RT-qPCR using Exiqon company design the miRNA primers.Bmf were amplified by RT-qPCR using the primers5'-3'TCCAAGAAGAGCCTCAGGAA and 5'-3'AAGAGAAAAAGCTGCCACCA The sequences of interest were transcribed into cDNA for the RT-qPCR reaction using the miRCURY LNA ${ }^{\mathrm{TM}}$ Universal RT microRNA PCR, SYBR Green master mix (Exiqon, Copenhagen, Denmark). The relative expression levels of gga-miR-221, gga-miR-222, and Bmf were calculated using the $2^{-\triangle \triangle C T}$ method.

\subsection{Data Analysis}

Data analysis was conducted using SPSS version 19.0 (IBM, Armonk, NY, USA). The homogeneity of variance was tested using the Levene's test of homogeneity of variance. If the variance was homogenous, Least-significant difference (LSD) inspection was carried out. If the variance was not homogenous, Dunnett's T3 inspection was performed. The Pearson linear correlation analysis was performed, and a $\mathrm{p}$ value less than 0.05 was established as the criterion for significance.

\subsection{Ethics Statement}

The above experiments were conducted in accordance with the institutional and national guidelines for the use and care of laboratory animals. The approval of using animals during the process of this study was obtained from South China Agricultural University Committee for Animal Experiments (approved ID: 201004152).

\subsection{Accession Number}

The microarray data were MIAME compliant which have been deposited in a MIAME compliant database (ArrayExpress, GEO ID: GSE28434). We have deposited the sequences of gga-miR-221 and gga-miR-222 (MI0001178, MI0001177) described in this paper in miRBase.

\section{Results}

\subsection{Expression of gga-miR-221 and gga-miR-222 in the Liver and Marrow of Pullets Infected with ALV-J}

Most of chickens in the ALV-J infected group gradually appeared in a comparatively emaciated and weak way by contrast with those of chickens in the control group. At 10 weeks, the size of livers of the infected chickens was obviously greater than that of the control group (Figure 1A). We first examined the expression levels of gga-miR-221 and gga-miR-222 in vivo in pullets infected with ALV-J. Gene chip analysis indicated that miR-221 and miR-222 were up-regulated significantly in the livers of infected pullets 10 days post infection (Figure 1B). QRT-PCR was used to confirm that the levels of gga-miR-221 were significantly up-regulated in the livers of the infected pullets compared to the control group at each time point examined, except for the 20-day-old time point $(p<0.01$; Figure $1 C)$. 
Likewise, qRT-PCR confirmed that gga-miR-222 levels were significantly increased in the infected group compared to the control group at each time point $(p<0.01$; Figure 1D).

\section{2. gga-miR-221 and gga-miR-222 Accelerate the Proliferation, Growth, and Migration of DF-1 Cells}

To understand the possible impacts of gga-miR-221 and gga-miR-222 on ALV-J tumorigenesis, we assessed the effects of over-expressing the miRNAs on the proliferation of DF- 1 cells over three days. Both gga-miR-221 and gga-miR-222 accelerated the proliferation of DF-1 cells compared to the NC group and the mock transfected cells (Figure 2A,B). There was no apparent difference in proliferation $24 \mathrm{~h}$ after transfection between the groups, but at $48 \mathrm{~h}$ after the transfection, the cells transfected with gga-miR-221 and gga-miR-222 had the highest levels of proliferation, which was confirmed by the colony formation assay (Figure 2C). The impacts of gga-miR-221 and gga-miR-222 on the growth and migration of DF-1 cells were assessed using an in vitro wound healing assay (Figure 2D). Differences in wound healing were apparent $24 \mathrm{~h}$ after the transfection, between the experimental groups, the NC group, and the mock transfected group. By $48 \mathrm{~h}$, there is a clear disparity between the experimental groups in which all the cells have completed the healing process and the other groups where there remains a visible growth gap between cells. These results provided additional support that gga-miR-221 and gga-miR-222 effect the growth and migration of DF-1 cells.

A

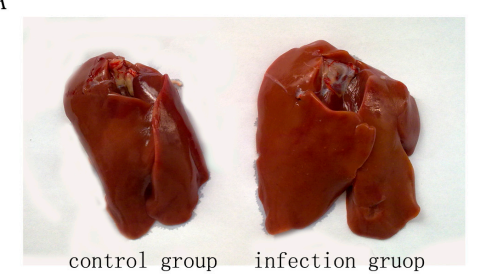

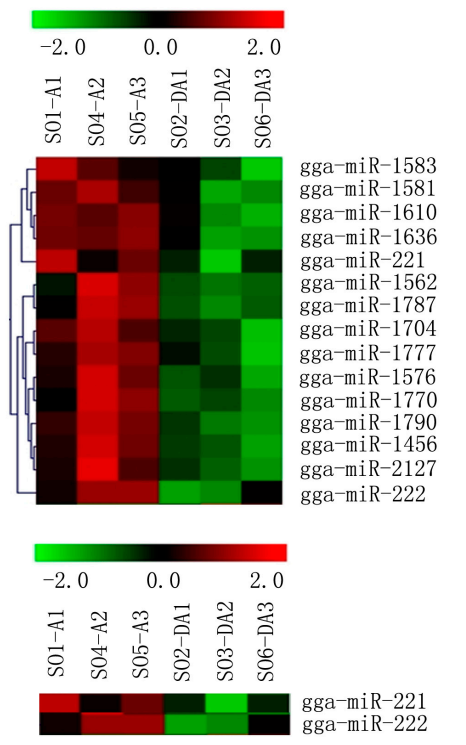

C

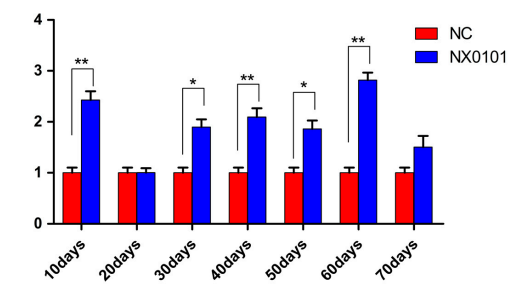

D

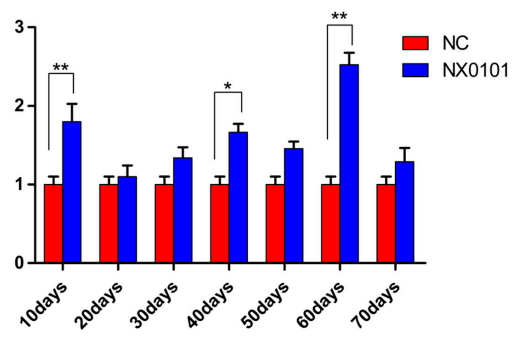

Figure 1. gga-miR-221 and gga-miR-222 expression was upregulated in ALV-J induced cancer. (A) Liver lesions in SPF white leghorn chickens induced by viral infection at 70 days; (B) Microarray analysis of miRNA expression during ALV-J infection. DA represents the control group and A represents tte infection group; (C) and (D) gga-miR-221 and gga-miR-222 expression levels in the liver of ALV-J infected chickens were quantified by qRT-PCR every 10 days from 10 to 70 days $\left({ }^{* *} p<0.01 ;{ }^{*} p<0.05\right)$. 
A

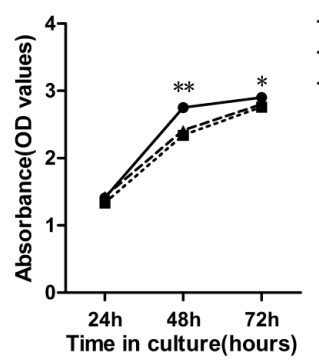

$\mathrm{C}$

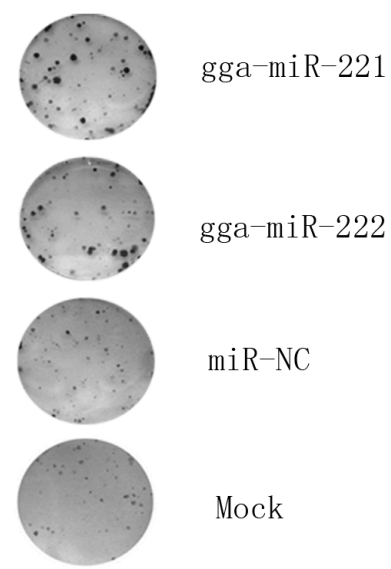

B

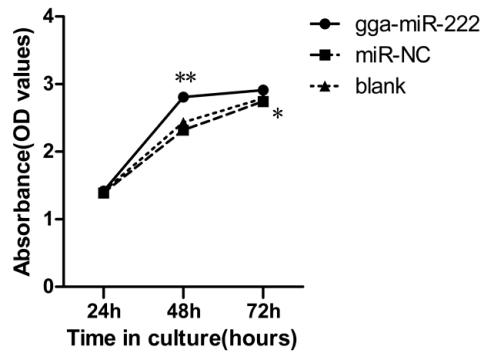

D

$0 \mathrm{~h}$
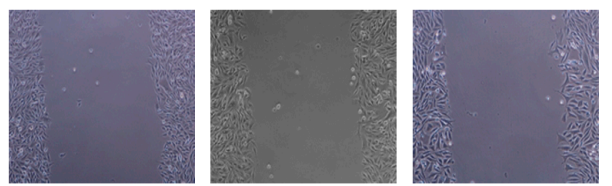

$24 \mathrm{~h}$
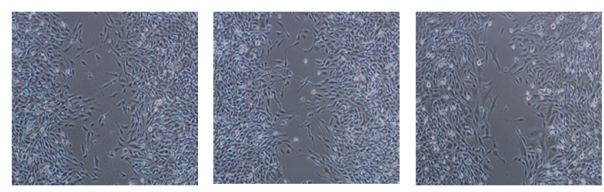

$48 \mathrm{~h}$
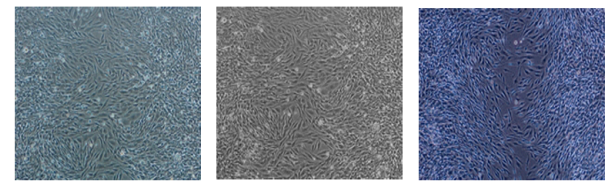

gga-miR-221 gga-miR-222 miR-NC

Figure 2. gga-miR-221 and gga-miR-222 promoted DF-1 cell proliferation and invasion. Cells were transfected with gga-miR-221, gga-miR-222, miR-NC, or mock transfected and then underwent WST-1 analysis, wound healing assay, and colony formation assay. (A) and (B) The effects of gga-miR-221 and gga-miR-222 on proliferation were detected using WST-1 analysis over $48 \mathrm{~h}$. The figures show the means and standard errors from three independent experiments; ${ }^{*} P$ value $(p) * * p<0.01$. (C) The effects of gga-miR-221 and gga-miR-222 on colony formation of DF-1 cells is shown. (D) $48 \mathrm{~h}$ after transfection, scratch wounds were made on confluent monolayer cultures. Images of the wound repair process were taken at 0,24 , and $48 \mathrm{~h}$ after wounding. Magnification is $200 \times$.

\section{3. gga-miR-221 and gga-miR-222 Inhibit Apoptosis}

DF-1 cells were transfected with gga-miR-221, gga-miR-222, NC miRNA, or mock transfected and then induced to undergo apoptosis using serum starvation $(48 \mathrm{~h})$, cisplatin treatment $(72 \mathrm{~h})$, or doxorubicin treatment $(72 \mathrm{~h})$. The level of apoptosis level in DF-1 cells that were transfected with gga-miR-221 and gga-miR-222 was substantially reduced compared to the NC groups and the control group, suggesting that the over-expression of gga-miR-221 and gga-miR-222 has anti-apoptotic effects (Figure 3A,B). The percentage of cells undergoing apoptosis and the stage of apoptosis were assessed by flow cytometry using Annexin V-FITC/PI staining. The percentage of cells undergoing apoptosis after transfection with gga-miR-221 was: $21 \%$ after serum starvation, $31.1 \%$ after cisplatin treatment, and $13.7 \%$ after doxorubicin treatment. Similarly, the percentage of cells transfected with gga-miR-222 undergoing apoptosis were: $18.8 \%$ after serum starvation, $25.1 \%$ after cisplatin treatment, and $14.8 \%$ after doxorubicin (Figure 3C). These results reaffirmed the anti-apoptotic effects of over-expressing gga-miR-221 and gga-miR-222. 

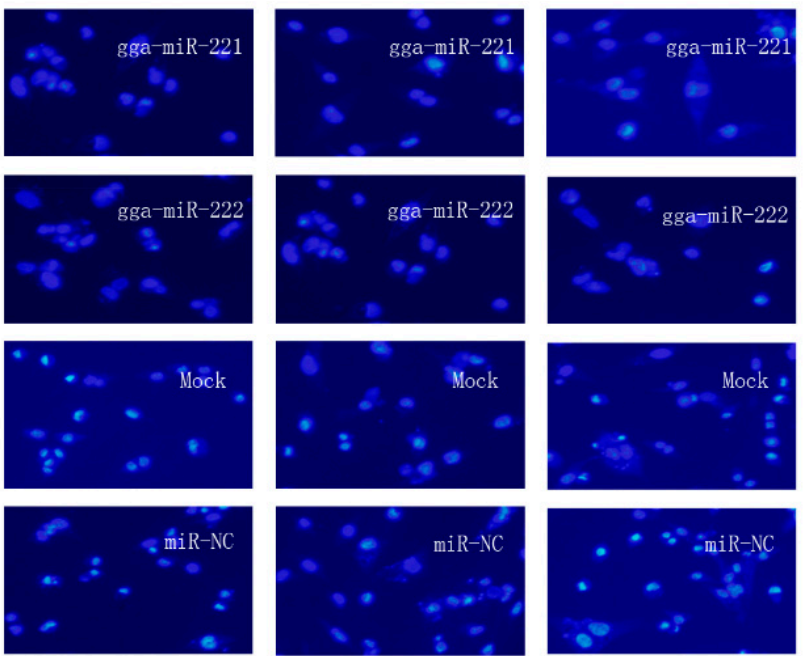

Serum-starvation $(72 \mathrm{~h})$

Cisplatin(48h)

Doxorubicin (48h)

B
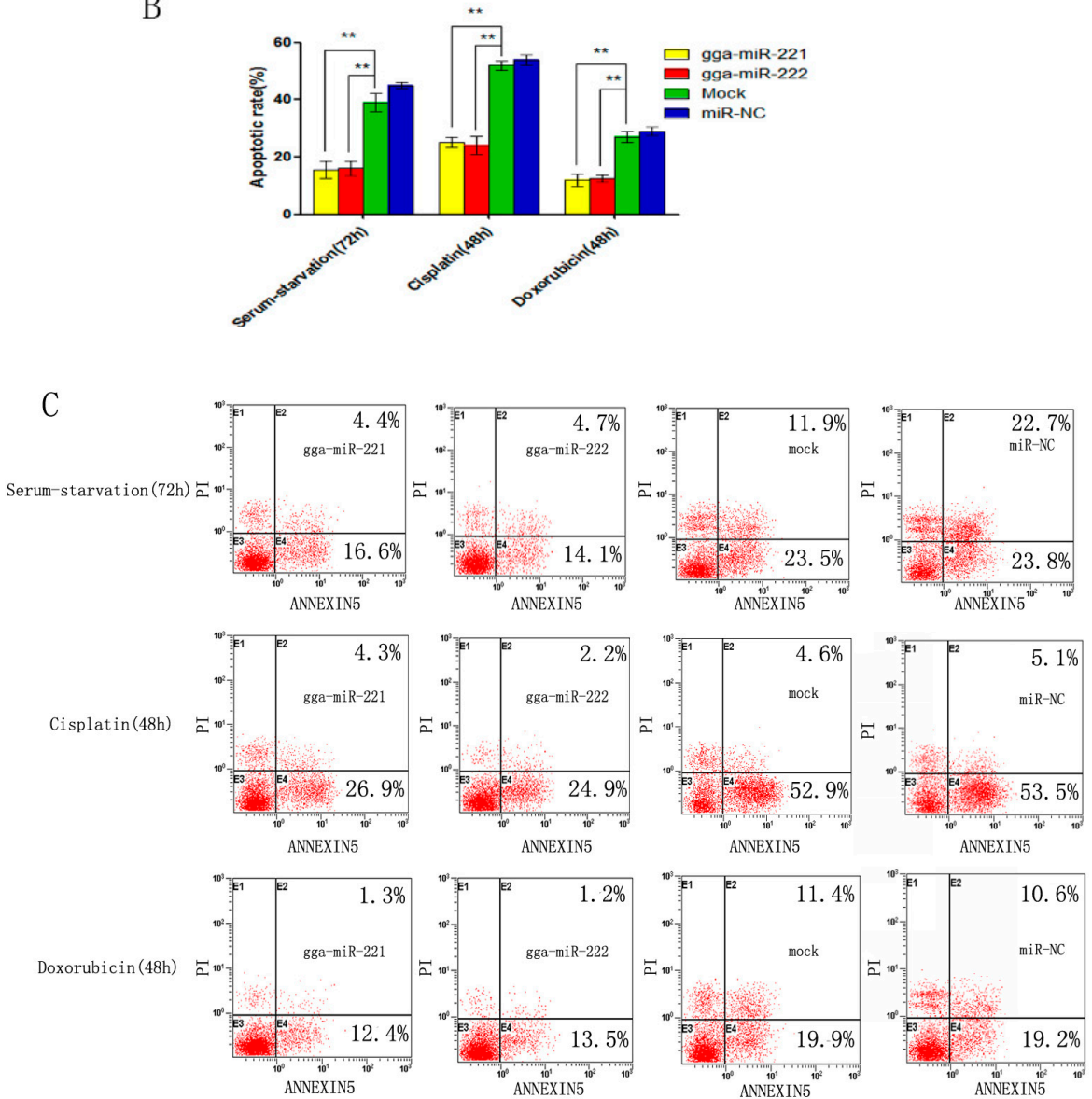

Figure 3. gga-miR-221 and gga-miR-222 inhibit apoptosis induced by serum starvation, cisplatin, and doxorubicin. Cells were transfected with gga-miR-221, gga-miR-222, miR-NC, or mock transfected and then stained with DAPI. (A) The rates of apoptosis were evaluated by assessing the apoptotic morphology of the cells. Magnification is $200 \times($ B) The differences in the rate of apoptosis between gga-miR-221, gga-miR-222, miR-NC, and mock transfected cells are shown. The graphs show the means and standard errors from three independent experiments ${ }^{* *} p<0.01$. (C) The cells transfected with gga-miR-221, gga-miR-222, miR-NC, or mock transfected and then stained with Annexin V-FITC/PI 48 or $72 \mathrm{~h}$ post-transfection. 
3.4. gga-miR-221 and gga-miR-222 Inhibit the Expression of BMF by Binding to the $3^{\prime}$-Untranslated Region (UTR)

The target genes of gga-miR-221 and gga-miR-222 were predicted utilizing online databases such as TargetScan, PicTar, and MicroCosm. RNAhybrid was used to increase the accuracy of the results through a functional analysis. The predicted results identified BMF as a target gene of gga-miR-221 and gga-miR-222 (Figure 4A). The gga-miR-221 is identical to Homo sapiens (hsa)-miR-221 and Mus musculus (muu)-miR-221 while the gga-miR-222 differs from hsa-miR-222 and muu-miR-222 by three bases (Figure 4B). The $3^{\prime}$ UTR sequence containing the miRNA binding sites was cloned into the reporter vector pmiR-GLO (BMF-3' UTR-wt). A mutant form of the BMF-3' UTR was also established (BMF-3' UTR-mut). The wild-type and mutant reporter vectors, and the miRNA and NC mimics transfected into DF- 1 cells. The miRNA constructs inhibited BMF-3' UTR-wt by approximately $50 \%$, but had no effect on expression on the BMF-3' UTR-mut construct $(p<0.01$; Figure 4C). DF-1 cells were transfected with gga-miR-221 and gga-miR-222 for $72 \mathrm{~h}$ and then the proteins were harvested for Western blot. The level of BMF protein in cells over-expressing gga-miR-221 and gga-miR-222 were reduced compared to the NC and control groups (Figure 4D), suggesting that over-expressed gga-miR-221 and gga-miR-222 inhibit the expression of BMF.

A

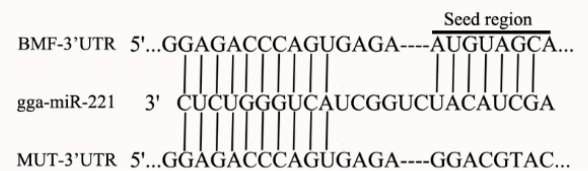

MUT-3'UTR 5'...GGAGACCCAGUGAGA----GGACGTAC...

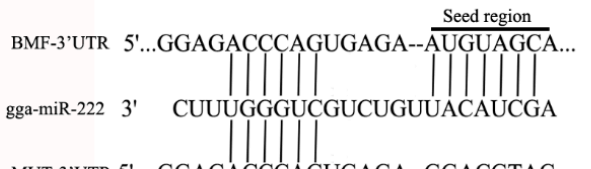

gga-miR-221 CTTTGGGTCGTCTGTTACATCGA hsa-miR-221 CTTTGGGTCGTCTGTTACATCGA muu-miR-221 CTTTGGGTCGTCTGTTACATCGA

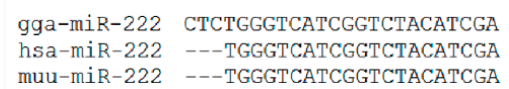

MUT-3'UTR 5'... GGAGACCCAGUGAGA--GGACGTAC...

B

$\mathrm{C}$
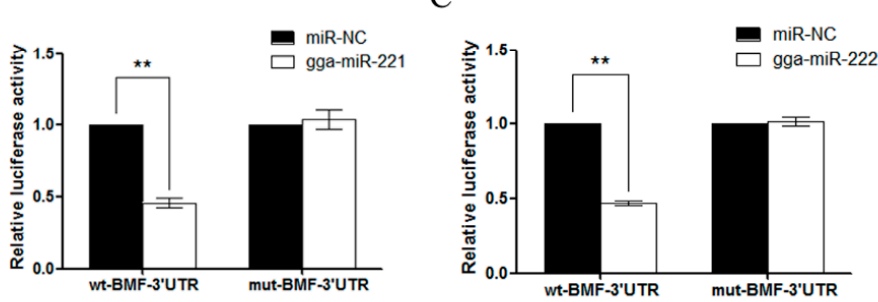

D

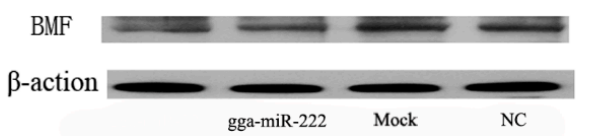

Figure 4. BMF is a direct target for gga-miR-221 and gga-miR-222. (A) Alignment of BMF-3'UTR, gga-miR-221,gga-miR-222 and MUT-3'UTR, where the complementary site for the seed region of gga-miR-221 and gga-miR-222 are indicated; (B) Differences in gga-miR-221/222, homo sapiens miR-221/222, and Mus musculus miR-221/222; (C) and (D) The regulation of luciferase activity from the BMF- $3^{\prime}$ UTR is dependent on gga-miR-221 and gga-miR-222. DF-1 cells were co-transfected with gga-miR-221, gga-miR-222, miR-NC and either wt-BMF-3' UTR (left) or mut-BMF-3' UTR (right). The graphs show the means and standard errors of at least three independent experiments. ${ }^{* *} p<0.01$, compared to miR-NC-transfected cells; (E) Ectopic expression of gga-miR-221 and gga-miR-222 reduced the level of BMF protein expression in DF-1 cells. $\beta$-action levels were used as a control. Each experiment was repeated three times, and each sample was assayed in triplicate. 


\subsection{The Expression of mRNA of BMF on Liver, Blood, and Spleen}

Given that gga-miR-221 and gga-miR-222 promote the proliferation, migration, and growth of DF-1 cells, and inhibit the apoptosis of DF-1 cells by suppressing the expression of BMF, we assessed the expression level of BMF mRNA in SPF chickens infected with ALV-J. In the liver, the level of BMF mRNA was downregulated 10 and 70 days after infection (Figure 5A). In the spleen, the level of BMF mRNA was downregulated 60 and 90 days after infection $(p<0.05$; Figure 5B). Finally, in the blood the level of BMF mRNA expression was downregulated 30, 40, and 90 days after infection $(p<0.05$; Figure 5C).

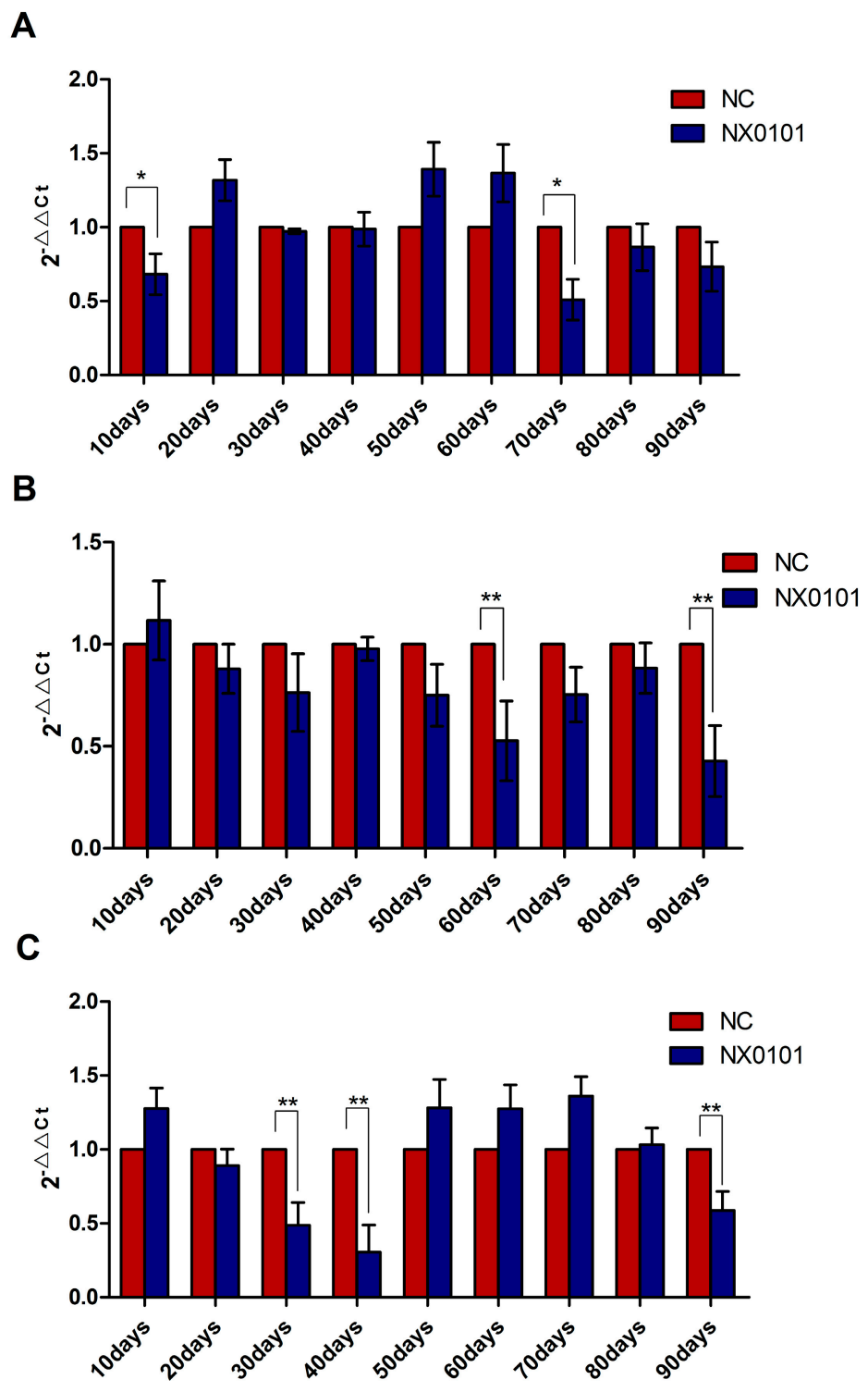

Figure 5. mRNA expression of BMF in the liver, spleen, and blood of chickens infected by ALV-J. (A) BMF mRNA expression levels in the liver of infected pullets from 10 to 90 days post infection. (B) BMF mRNA expression levels in the spleen of infected pullets from 10 to 90 days post infection. (C) BMF mRNA expression levels in the blood of infected pullets from 10 to 90 days post infection. ** $p<0.01, * p<0.05$. 


\section{Discussion}

In this study, we provide evidence that gga-miR-221 and gga-miR-222 are up-regulated in the livers of pullets infected with ALV-J. Furthermore, we demonstrate that gga-miR-221 and gga-miR-222 have stimulating effects on cell growth and proliferation, and that BMF is a likely target for gga-miR-221 and gga-miR-222. Taken together, our results and studies in the Marek's disease virus (MDV) model suggest that up-regulation of gga-miR-221 and gga-miR-222 might be a general feature of oncogenic poultry viruses.

To better understand the anti-apoptotic function of gga-miR-221 and gga-miR-222, apoptosis was induced using three different methods, serum starvation, cisplatin treatment, and doxorubicin treatment. It is well established that tumor cell proliferation exceeds the limitations of normal cells, and because of their rapid growth, they are constantly challenged by nutrient deficiency. Thus, understanding the anti-apoptotic capabilities of tumor cells under conditions of malnutrition is critical. Serum starved DF-1 cells that were transfected with gga-miR-221 and gga-miR-222 displayed relatively low rates of apoptosis, confirming that gga-miR-221 and gga-miR-222 are anti-apoptotic when cells are malnourished. Cisplatin, a broad-spectrum chemotherapeutic, remains in widespread clinical use. It inhibits tumor cell DNA replication and destroys the structure of the cell membrane resulting in apoptosis. In our study, cells transfected with gga-miR-221 and gga-miR-222 were relatively resistant to cisplatin-induced apoptosis. Similarly, cells transfected with gga-miR-221 and gga-miR-222 were resistant to doxorubicin-induced apoptosis.

Over-expression of miR-221 is associated with a more aggressive phenotype of hepatocellular carcinoma and inhibits apoptosis by binding to BMF. In addition to its own pro-apoptotic function, the BMF gene can regulate the pro-apoptotic function of BCL-2 by binding to it [40]. As it has been confirmed as the target gene of gga-miR-221/222 in our experiments. The qRT-PCR data showed that BMF was down-regulated in the spleen at each time point assessed, and was downregulated in the liver and blood during the later period of infection, which may contribute to ALV-J developing into a chronic disease.

MDV is well studied by scholars in the field as it has caused many cases of acute tumor diseases. Unlike ALV-J, MDV contains a cancer gene in its genome and miRNAs that promote the development of cancer. MDV causes several types of cancer in different organs; and differential expression of miRNAs during MDV infection have been reported [41,42]. Distinct from MDV and other viruses that contain a proto-oncogene, The non-oncogene-bearing retrovirus causes tumors by inserting into the host gene resulting in an insertional mutation [43]. During the lasting period of incubation, cumulative insertional mutations stimulate traditional proto-oncogenes and triggers miRNA dysfunction [44]. Therefore, analyses to detect the expression of miRNAs and genes could possibly elucidate the tumorigenic mechanisms of ALV-J. Taken together, our results suggest that miR-221 and miR-222 regulate BMF and inhibit apoptosis through the mitochondrial pathway. Thus, by regulating miR-221 and miR-222, ALV-J, promotes the proliferation, migration, and growth of cells, and inhibits apoptosis to induce tumor formation.

Acknowledgments: We want to take this opportunity to extend our great appreciation towards Zhizhong Cui from Shandong Agricultural University for his kind offer of the NX0101 strain of ALV-J for this study. Also, this work wouldn't have been accomplished without supports and grants from the National Natural Science Foundation of China $(31072152,31472217)$ and the Open Project of the State Key Laboratory of Biocontrol (SKLBC2010K07).

Author Contributions: Jun Ji and Hongxin Li conceived and designed research. Zhenkai Dai, Jun Ji and Yiming Yan performed the experiments. Feng Chen and Wencheng Lin analyzed the data. Weiguo Chen, Yingzuo Bi, and Qingmei Xie contributed materials/analysis tools; Zhenkai Dai and Yang liu wrote the manuscript. Both authors reviewed the manuscript.

Conflicts of Interest: The authors declare no conflicts of interest. 


\section{References}

1. Payne, L.N.; Nair, V. The long view: 40 Years of avian leukosis research. Avian Pathol. 2012, 41, 11-19. [CrossRef] [PubMed]

2. Ji, J.; Li, H.; Zhang, H.; Xie, Q.; Chang, S.; Shang, H.; Ma, J.; Bi, Y. Complete genome sequence of an avian leukosis virus isolate associated with hemangioma and myeloid leukosis in egg-type and meat-type chickens. J. Virol. 2012, 86, 10907-10908. [CrossRef] [PubMed]

3. Li, H.; Xue, C.; Ji, J.; Chang, S.; Shang, H.; Zhang, L.; Ma, J.; Bi, Y.; Xie, Q. Complete genome sequence of a J subgroup avian leukosis virus isolated from local commercial broilers. J. Virol. 2012, 86, 11937-11938. [CrossRef] [PubMed]

4. Neumann, U. Morphological evidence for avian leukosis virus (ALV) subgroup j avian myelocytomatosis observed in broiler parent flocks in Costa Rica. In Proceedings of the International Symposium on ALV-J and other Avian Retroviruses, Rauischolzhauzen, Germany, 5-8 June 2000; pp. 181-183.

5. Bagust, T.J. Avian leukosis virus-J in Australia: Scenarios and options for control. In Proceedings of the International Symposium on ALV-J and Other Avian Retroviruses, Rauischolzhauzen, Germany, 5-8 June 2000; pp. 234-239.

6. Bagust, T.J.; Fenton, S.P.; Reddy, M.R. Detection of subgroup j avian leukosis virus infection in Australian meat-type chickens. Aust. Vet. J. 2004, 82, 701-706. [CrossRef] [PubMed]

7. Otsuka, K.; Ochiya, T. Genetic networks lead and follow tumor development: MicroRNA regulation of cell cycle and apoptosis in the p53 pathways. Biomed. Res. Int. 2014, 2014, 749724. [CrossRef] [PubMed]

8. Nicholson, D.W.; Thornberry, N.A. Apoptosis:Life and death decisions. Science 2014, 299, 214-215. [CrossRef] [PubMed]

9. Antonsson, B. Bax and other pro-apoptotic Bcl-2 family "Killer-Proteins" and their victim the mitochondrion. Cell Tissue Res. 2001, 306, 347-361. [CrossRef] [PubMed]

10. Cepero, E.; Johnson, B.W.; Boise, L.H. Cloning and analysis of Bcl-2 family genes. Methods Cell Biol. 2001, 66, 29-47. [PubMed]

11. Weerasinghe, P.; Hallock, S.; Tang, S.; Liepins, A. Role of Bcl-2 family proteins and Caspase-3 in sanguinarine-induced bimodal cell death. Cell Biol. Toxicol. 2001, 17, 371-381. [CrossRef] [PubMed]

12. Zhang, C.; Zhang, J.; Zhang, A.; Wang, Y.; Han, L.; You, Y.; Pu, P.; Kang, C. PUMA is a novel target of miR-221/222 in human epithelial cancers. Int. J. Oncol. 2010, 37, 1621-1626. [PubMed]

13. Marzo, I.; Brenner, C.; Zamzami, N.; Jürgensmeier, J.M.; Susin, S.A.; Vieira, H.L.; Prévost, M.C.; Xie, Z.; Matsuyama, S.; Reed, J.C.; et al. Bax and adenine nucleotide translocator cooperate in the mitochondrial control of apoptosis. Science 1998, 281, 2027-2031. [CrossRef] [PubMed]

14. Kim, H.; Tu, H.C.; Ren, D.; Takeuchi, O.; Jeffers, J.R.; Zambetti, G.P.; Hsieh, J.J.; Cheng, E.H. Stepwise Activation of BAX and BAK by tBID, BIM, and PUMA Initiates Mitochondrial Apoptosis. Mol. Cell 2009, 36, 487-499. [CrossRef] [PubMed]

15. Ren, D.; Tu, H.C.; Kim, H.; Wang, G.X.; Bean, G.R.; Takeuchi, O.; Jeffers, J.R.; Zambetti, G.P.; Hsieh, J.J.; Cheng, E.H. BID, BIM, and PUMA are essential for activation of the BAX and BAK-dependent cell death program. Science 2010, 330, 1390-1393. [CrossRef] [PubMed]

16. Letai, A. Puma Strikes Bax. J. Cell Biol. 2009, 185, 189-191. [CrossRef] [PubMed]

17. Chen, L.; Willis, S.N.; Wei, A.; Smith, B.J.; Fletcher, J.I.; Hinds, M.G.; Colman, P.M.; Day, C.L.; Adams, J.M.; Huang, D.C. Differential targeting of prosurvival Bcl-2 proteins by their BH3-only ligands allows complementary apoptotic function. Mol. Cell 2005, 17, 393-403. [CrossRef] [PubMed]

18. Gérecová, G.; Kopanicová, J.; Jaká, P.; Běhalová, L.; Juhásová, B.; Bhatia-Kiššová, I.; Forte, M.; Polčic, P.; Mentel, M. BH3-only proteins Noxa, Bik, Bmf, and Bid activate Bax and Bak indirectly when studied in yeast model. FEMS Yeast Res. 2013, 13, 747-754. [CrossRef] [PubMed]

19. Gomez-Esquer, F.; Palomar, M.A.; Rivas, I.; Delcan, J.; Linares, R.; Diaz-Gil, G. Characterization of the BH3 protein Bmf in Gallus gallus: Identification of a novel chicken-specific isoform. Gene 2008, 407, $21-29$. [CrossRef] [PubMed]

20. Egle, A.; Harris, A.W.; Bouillet, P.; Cory, S. Bim is a suppressor of Myc-induced mouse B cell leukemia. Proc. Natl. Acad. Sci. USA 2004, 101, 6164-6169. [CrossRef] [PubMed] 
21. Tagawa, H.; Karnan, S.; Suzuki, R.; Matsuo, K.; Zhang, X.; Ota, A.; Morishima, Y.; Nakamura, S.; Seto, M. Genome-wide array-based CGH for mantle cell lymphoma: Identification of homozygous deletions of the proapoptotic gene BIM. Oncogene 2005, 24, 1348-1358. [CrossRef] [PubMed]

22. Bartel, D.P. MicroRNAs: Genomics, biogenesis, mechanism, and function. Cell 2004, 116, 281-297. [CrossRef]

23. Shukla, G.C.; Singh, J.; Barik, S. MicroRNAs: Processing, maturation, target recognition and regulatory functions. Mol. Cell Pharmacol. 2011, 3, 83-92. [PubMed]

24. Calin, G.A.; Dumitru, C.D.; Shimizu, M.; Bichi, R.; Zupo, S.; Noch, E.; Aldler, H.; Rattan, S.; Keating, M.; Rai, K.; et al. Frequent deletions and down-regulation of micro-RNA genes miR15 and miR16 at 13Q14 in chronic lymphocytic leukemia. Proc. Natl. Acad. Sci. USA 2002, 99, 15524-15529. [CrossRef] [PubMed]

25. Cimmino, A.; Calin, G.A.; Fabbri, M.; Iorio, M.V.; Ferracin, M.; Shimizu, M.; Wojcik, S.E.; Aqeilan, R.I.; Zupo, S.; Dono, M.; et al. MiR-15 and miR-16 induce apoptosis by targeting BCL2. Proc. Natl. Acad. Sci. USA 2005, 102, 13944-13949. [CrossRef] [PubMed]

26. Lee, E.F.; Dewson, G.; Evangelista, M.; Pettikiriarachchi, A.; Gold, G.J.; Zhu, H.; Colman, P.M.; Fairlie, W.D. The functional differences between pro-survival and pro-apoptotic B cell lymphoma 2 (Bcl-2) proteins depend on structural differences in their Bcl-2 Homology 3 (BH3) domains. J. Biol. Chem. 2014, 289, 36001-36017. [CrossRef] [PubMed]

27. Li, H.; Shang, H.; Shu, D.; Zhang, H.; Ji, J.; Sun, B.; Xie, Q. Gga-miR-375 plays a key role in tumorigenesis post subgroup J avian leukosis virus infection. PLoS ONE 2014, 9. [CrossRef] [PubMed]

28. Garofalo, M.; Quintavalle, C.; Romano, G.; Croce, C.M.; Condorelli, G. MiR221/222 in cancer: Their role in tumor progression and response to therapy. Curr. Mol. Med. 2012, 12, 27-33. [CrossRef] [PubMed]

29. Li, H.; Ji, J.; Xie, Q.; Shang, H.; Zhang, H.; Xin, X.; Chen, F.; Sun, B.; Xue, C.; Ma, J. Aberrant expression of liver micoRNA in chickens infected with subgroup J avian leukosis virus. Virus Res. 2012, 169, $268-271$. [CrossRef] [PubMed]

30. Frenquelli, M.; Muzio, M.; Scielzo, C.; Fazi, C.; Scarfò, L.; Rossi, C.; Ferrari, G.; Ghia, P.; Caligaris-Cappio, F. MicroRNA and proliferation control in chronic lymphocytic leukemia: Functional relationship between miR-221/222 cluster and P27. Blood 2010, 115, 3949-3959. [CrossRef] [PubMed]

31. Rommer, A.; Steinleitner, K.; Hackl, H.; Schneckenleithner, C.; Engelmann, M.; Scheideler, M.; Vlatkovic, I.; Kralovics, R.; Cerny-Reiterer, S.; Valent, P.; et al. Overexpression of primary microRNA 221/222 in acute myeloid leukemia. BMC Cancer 2013, 13, 364. [CrossRef] [PubMed]

32. Miller, T.E.; Ghoshal, K.; Ramaswamy, B.; Roy, S.; Datta, J.; Shapiro, C.L.; Vlatkovic, I.; Kralovics, R.; Cerny-Reiterer, S.; Valent, P.; et al. MicroRNA-221/222 confers tamoxifen resistance in breast cancer by targeting p27Kip1. J. Biol. Chem. 2008, 283, 29897-29903. [CrossRef] [PubMed]

33. Lin, D.; Cui, F.; Bu, Q.; Yan, C. The expression and clinical significance of GTP-binding RAS-like 3 (ARHI) and microRNA 221 and 222 in prostate cancer. J. Int. Med. Res. 2011, 39, 1870-1875. [CrossRef] [PubMed]

34. Chun-Zhi, Z.; Lei, H.; An-Ling, Z.; Yan-Chao, F.; Xiao, Y.; Guang-Xiu, W.; Zhi-Fan, J.; Pei-Yu, P.; Qing-Yu, Z.; Chun-Sheng, K. MicroRNA-221 and microRNA-222 regulate gastric carcinoma cell proliferation and radioresistance by targeting PTEN. BMC Cancer 2010, 10. [CrossRef]

35. Galardi, S.; Mercatelli, N.; Giorda, E.; Massalini, S.; Frajese, G.V.; Ciafrè, S.A.; Farace, M.G. MiR-221 and miR-222 expression affects the proliferation potential of human prostate carcinoma cell lines by targeting p27Kip1. J. Biol. Chem. 2007, 282, 23716-23724. [CrossRef] [PubMed]

36. Felli, N.; Fontana, L.; Pelosi, E.; Botta, R.; Bonci, D.; Facchiano, F.; Liuzzi, F.; Lulli, V.; Morsilli, O.; Peschle, C.; et al. MicroRNAs 221 and 222 inhibit normal erythropoiesis and erythroleukemic cell growth via kit receptor down-modulation. Proc. Natl. Acad. Sci. USA 2005, 13, 18081-18086. [CrossRef] [PubMed]

37. Gramantieri, L.; Fornari, F.; Ferracin, M.; Veronese, A.; Sabbioni, S.; Calin, G.A.; Grazi, G.L.; Croce, C.M.; Bolondi, L.; Negrini, M. MicroRNA-221 targets Bmf in hepatocellular carcinoma and correlates with tumor multifocality. Clin. Cancer Res. 2009, 15, 5073-5081. [CrossRef] [PubMed]

38. Lambeth, L.S.; Yao, Y.; Smith, L.P.; Zhao, Y.; Nair, V. MicroRNAs 221 and 222 Target p27Kip1 in Marek's disease virus-transformed tumour cell line MSB-1. J. Gen. Virol. 2009, 90, 1164-1171. [CrossRef] [PubMed]

39. Cui, Z.; Sun, S.; Wang, J. Reduced serologic response to newcastle disease virus in broiler chickens exposed to a Chinese field strain of subgroup J avian leukosis virus. Avian Dis. 2006, 50, 191-195. [CrossRef] [PubMed]

40. Kepp, O.; Gottschalk, K.; Churin, Y.; Rajalingam, K.; Brinkmann, V.; Machuy, N.; Kroemer, G.; Rudel, T. Bim and Bmf synergize to induce apoptosis in neisseria gonorrhoeae infection. PLoS Pathog. 2009, 3, e1000348. [CrossRef] [PubMed] 
41. Luo, J.; Sun, A.J.; Teng, M.; Zhou, H.; Cui, Z.Z.; Qu, L.H.; Zhang, G.P. Expression profiles of microRNAs encoded by the oncogenic Marek's Disease virus reveal two distinct expression patterns in vivo during different phases of disease. J. Gen. Virol. 2011, 92, 608-620. [CrossRef] [PubMed]

42. Tian, F.; Luo, J.; Zhang, H.; Chang, S.; Song, J. MiRNA expression signatures induced by Marek's disease virus infection in chickens. Genomics 2012, 99, 152-159. [CrossRef] [PubMed]

43. Dabrowska, M.J.; Dybkaer, K.; Johnsen, H.E. Loss of MicroRNA targets in the 3' untranslated region as a mechanism of retroviral insertional activation of growth factor independence 1. J. Virol. 2009, 83, 8051-8061. [CrossRef] [PubMed]

44. Chesters, P.M.; Howes, K.; McKay, J.C.; Payne, L.N.; Venugopal, K. Acutely transforming avian leukosis virus subgroup J strain 966: Defective genome encodes a 72-kilodalton Gag-Myc fusion protein. J. Virol. 2001, 75, 4219-4225. [CrossRef] [PubMed]

(C) 2015 by the authors; licensee MDPI, Basel, Switzerland. This article is an open access article distributed under the terms and conditions of the Creative Commons by Attribution (CC-BY) license (http://creativecommons.org/licenses/by/4.0/). 